Methods A hospital-based cross sectional descriptive study was conducted in Nay Pyi Taw with the aim of exploring the road user pattern, factors related to motorcycle accident and outcomes of accident among 171 motorcyclists, passengers and pedestrians admitted to two hospitals. Face to face interviews were conducted by using pre-tested questionnaire with motorcycle accident patients and their attendants.

Results Male, under 30 year age group, middle school level education and sale workers were most common victims. Motorcyclists were most at risk road users for accidents (57\%) and 40\% was due to fall or slipped motorcycles. Driving without licenses (31\%), drinking alcohol (19\%), high speeding (21\%) and overtracking (3.5\%), impaired visibility (9.4\%) and broken break or tires or engines $(4 \%)$ were associated with accidents. Forty eight percent of urban accident occurred on the straight roads where as $16 \%$ and $6 \%$ of rural accident occurred on rough and curved roads. Mortality rate was $9.9 \%$ whereas $4 \%$ was brought in dead cases. Nineteen percent treated as out-patients, 53\% as nonsevere in-patients and $18 \%$ was severe-in patients. Head injury and fracture in lower limbs were most common $36.3 \%$ and $30.4 \%$ respectively). Dead cases were due to head injury $(76.5 \%)$ and multiple injuries $(23.5 \%)$. Only $18 \%$ received first aids emergency treatment and $4.5 \%$ was sent to hospital with ambulance. Median risk cores of human factors, protective factors, vehicle factors and environmental factors were significantly higher on rural cases, pedestrian, severe in-patients and dead cases.

Conclusion Findings informed to responsible authorities for road traffic laws enforcement, intervention program development, emergency and medical services to reduce morbidity and mortality among road traffic injury patients.

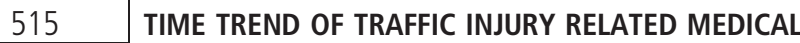 UTILISATION AND EXPENDITURES IN TAIWAN FROM $2003-2012$}

Ping-Ling Chen. Taipei Medical University, Taiwan

10.1136/injuryprev-2016-042156.515

Background Traffic injury is the leading external cause of unintentional injury in Taiwan. The aim of this study was to determine the utilisation of emergency-visit, hospitalisation, and medical expenditures by different type of vehicle crash.

Methods Claim data were collected from the Taiwan National Health Insurance Research Database for patients who were identified from the Taiwan National Traffic Accident Dataset from 2003 to 2012. Measurements included type of medical utilisation, medical expenditures, and type of vehicle crash. Frequency counts, percentages and 95\% confident interval were estimated for descriptive analyses. Mann-Kendall test was used to determine time trend of medical utilisation and medical expenditures by car types.

Results Over the period 2003 to 2012, the results of time trend test showed that the percentage of hospitalisation due to traffic injury had significantly decreased with years, however, the ERvisit utilisation increased from $27.4 \%$ in 2003 to $34.2 \%$ in 2012. In addition, both of the total medical expenditure of ER-visit and hospitalisation had significantly increased by years. In 2012, the total medical expenditure of ER-visit, hospitalisation was over 16.6 million US dollars and 47.4 million US dollars respectively. After stratification of car types, the ER-visit utilisation, total ER expenditure and total hospitalisation expenditure among twowheeler drivers remained significantly increased by years.
Although the ER-visit and medical utilisation among four-wheeler drivers was significantly decreased, the total medical expenditure of ER-visit among four-wheeler drivers had been significantly increased

Conclusions Effective traffic injury prevention programs are needed to develop for reducing the burden of public health and medical services.

\section{CHILDREN ARE WALKING! INNOVATIVE STUDY OF TRAVEL PATTERNS AND SAFETY}

${ }^{1}$ Sharon Levi, ${ }^{2}$ David Zaidel, ${ }^{3}$ Victoria Gitelman, ${ }^{4}$ Ido Prihed, ${ }^{1}$ Orly Silbinger. ${ }^{1}$ Beterem- Safe Kids Israel; ${ }^{2}$ Sight Ergonomics and Safety; ${ }^{3}$ Ran Naor Centrefor Road Safety Research, Technion; ${ }^{4}$ Prihed Geography and Survey Company

\subsection{6/injuryprev-2016-042156.516}

Background An increasing interest in the lifestyle and active mobility of children and continuing concern for their travel safety and secure environment requires detailed information about trip patterns and activities. There is little research on travel patterns of children, in particular during leisure hours. This study was designed to demonstrate child travel patterns and safety in Israel utilising advanced trip survey tools and methods.

Methods Phase 1 included a national self-report internet based travel survey as well as an innovative GPS based travel survey and follow up CAPI interview in Jerusalem with children age 612. Phase 2 included an in-depth study of pedestrian behaviour based on video analysis at major intersections and GPS based travel survey using cellular phones in three cities in central Israel. Results The findings indicate that walking is the most common mode of travel and children in Israel walk at a higher rate than their counterparts in other developed countries. The results include distances travelled, travel purposes and destinations, and differences among population groups. The study points to a number of risk-taking behaviours, including: dangerous walking while accompanied by an adult; vehicles not yielding to children at crosswalks; bicycling on roads and without helmets from a young age; and lack of safe pedestrian walkways.

Conclusions It is important to increase active mobility as part of a healthy lifestyle, however more efforts are needed to determine dangers to child pedestrians and promote safe walking. The study demonstrated feasibility of a GPS tracking method as well as the ability to obtain reliable activity and trip information from children as young as six years. The GIS spatial analysis provides an interesting and new perspective on child mobility and can influence both urban design and public health programming by identifying specific areas for behavioural and environmental treatment.

\section{THE CHALLENGES OF ENFORCING ROAD SAFETY POLICIES}

Gayle Di Pietro, Cristina Inclan, Cessie Petchi. Global Road Safety Partnership (GRSP)

\subsection{6/injuryprev-2016-042156.517}

Background Strong road safety policies that are strategically and effectively enforced optimise the safety of road users. In road safety, those responsible for enforcing the law are usually road police. In low and middle income countries, road police face many challenges as they are usually under-resourced and poorly trained to do the job of enforcement. 